\title{
BMJ Open Protocol for a scoping review about ethics in transition programmes for adolescents and young adults with neurodisabilities
}

\author{
Aline Bogossian, ${ }^{1,2}$ Jan Willem Gorter, ${ }^{3,4}$ Eric Racine ${ }^{1,5,6}$
}

To cite: Bogossian A, Gorter JW, Racine E. Protocol for a scoping review about ethics in transition programmes for adolescents and young adults with neurodisabilities. BMJ Open 2018:8:e020914. doi:10.1136/ bmjopen-2017-020914

- Prepublication history and additional material for this paper are available online. To view these files, please visit the journal online (http://dx.doi. org/10.1136/bmjopen-2017020914).

Received 30 November 2017

Accepted 3 July 2018
Revised 12 June 2018

\section{ABSTRACT}

Introduction As adolescents with neurodisabilities near adulthood, they and their caregivers will face the challenge of transferring from paediatric to adult healthcare systems. Despite the growing number of programmes designed to support healthcare transitions, little is known about practices that result in positive outcomes or how ethical issues are addressed. The objective of this review is to identify and document the ethical principles that may be embedded in the vision of transitional care programmes and to map ethical issues encountered in transitional care as well as the strategies employed to recognise and address these ethical issues.

Method and analysis We will document hidden and explicit ethical principles and issues from empirical studies on transition programmes for youth with neurodisabilities. An initial framework developed through a content extraction strategy will serve as a point of departure for data analysis and will be iteratively refined through our analytic process and with feedback from our stakeholders, including youth with neurodisabilities and their families. Findings will then be shared in different formats with health and social services professionals, healthcare decision and policymakers.

Dissemination Scoping review results will be shared at key conferences and disseminated in peer-reviewed publications as well as plain language summaries with stakeholders and the general public. Opportunities will be sought to discuss this project and its preliminary findings with partners in the paediatric, adult healthcare and rehabilitation communities comprising young people with neurodisabilities, their families, clinicians, programme managers and researchers.

\section{INTRODUCTION}

(C) Author(s) (or their employer(s)) 2018. Re-use permitted under CC BY-NC. No commercial re-use. See rights and permissions. Published by BMJ.

For numbered affiliations see end of article.

Correspondence to

Dr. Eric Racine;

eric.racine@ircm.qc.ca
As they grow up and become adults, adolescents with neurodisabilities (such as cerebral palsy, autism, Duchenne muscular dystrophy or other conditions that impact the neurological or neuromuscular systems) and their parents and caregivers will face the challenge of transferring from paediatric to adult healthcare systems. ${ }^{1}{ }^{2}$ In this scoping review, we adopt a definition of neurodisabilities developed through consultation with

\section{Strengths and limitations of this study}

- Repository of ethical visions and issues encountered in transition programmes for young adults with neurodisabilities.

- Informed by empirical literature as well as stakeholder engagement.

No quality assessment of studies.

a multidisciplinary group of health professionals and parents of children with neurological conditions in our selection of studies. ${ }^{3}$ Healthcare transition has been defined as the 'purposeful, planned movement of adolescents and young adults with chronic physical and medical conditions from child-centred to adult-oriented healthcare systems that is uninterrupted, coordinated, developmentally appropriate, psychosocially sound and comprehensive. ${ }^{4}$ Despite the growth of healthcare transition programmes across paediatric specialties, little is known about 'transition practices that produce positive outcomes ${ }^{56}$ in general, and more specifically, for youth with neurodisabilities. ${ }^{7}$

There is an increased interest in and necessity to evaluate whether transition programmes ensure a seamless transfer from paediatric to adult healthcare systems. ${ }^{589}$ For example, little is known about how professionals working in transitional care programmes seek to understand the values of young adults and their caregivers, place importance on

\footnotetext{
${ }^{\mathrm{i}}$ Definition of neurodisability: neurodisability describes a group of congenital or acquired long-term conditions that are attributed to impairment of the brain and/or neuromuscular system and create functional limitations. A specific diagnosis may not be identified. Conditions may vary over time, occur alone or in combination and include a broad range of severity and complexity. The impact may include difficulties with movement, cognition, hearing and vision, communication, emotion and behaviour.
} 


\section{Anticipatory \\ and Abstract}

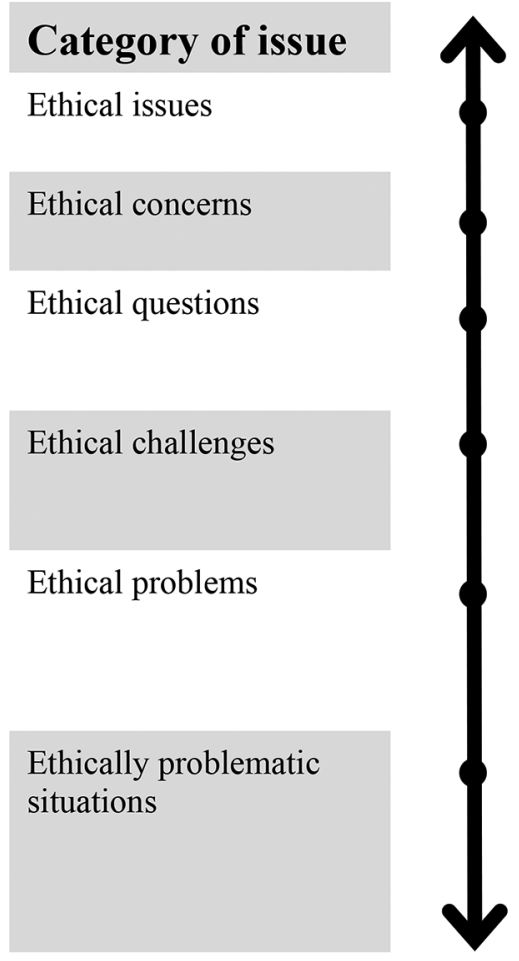

\section{Realized and Concrete}

Figure 1 Conceptualisation of categories of ethical issues.

\section{Description}

A potential stake that needs discussion

A worrisome stake that needs discussion and attention

A worrisome stake that needs discussion, attention and be answered

A worrisome stake that needs discussion, attention and be answered and surmounted

A worrisome stake that needs discussion, attention and be answered and surmounted via a response

A worrisome stake that needs discussion, attention and be answered, surmounted via a concrete response taking into account specific agents and context involved

\section{Example}

Confidentiality

Confidentiality in discussing sensitive information with parents

Should confidential information be discussed with parents?

How can I surmount the challenge of discussing confidential information with parents?

How can I resolve the problem of sharing confidential information with parents?

How can I resolve the problem of sharing confidential information with parents in this concrete situation composed of specific agents and a context? understanding their interdependence and ensure that their needs are integrated, developed and supported, in order to facilitate a smooth transition, ${ }^{10}$ or whether and how the assessment of patient values and preferences are integrated in healthcare transition programmes. ${ }^{7}$ The importance of recognising ethical issues that emerge in the clinical care of adolescents and young adults with neurodisabilities has been identified as an imperative to achieve purposeful practice goals such as the development of autonomy and the preparation of youth and their caregivers for decision-making. ${ }^{11}$

In the proposed scoping review, we will identify ethical principles that may be embedded in the vision of transition programmes and will map ethical issues that have been encountered in transitional care programmes. In our review, we will focus on documenting practices that support autonomy, respect preferences and capacities and facilitate new trusting relationships in the adult system. ${ }^{7}$ These latter issues can become particularly challenging for youth with significant impairments, dual diagnoses and complex care needs.

In this review, we conceptualise ethical issues on a continuum, spanning from ethically problematic situations that have occurred in practice and are documented to more abstract ethical issues that are anticipatory in nature (see figure 1 for categories of ethical issues).
The literature documenting ethical issues and visions in healthcare transitions is small, yet suggests research questions. Among those are (A) issues related to the gradual transfer of responsibility from parents/healthcare providers to youth and (B) explorations about how autonomy is developed in a way that is both empowering and satisfies and respects the capacities and preferences of youth. ${ }^{7}$ In exploring the ethical visions and issues in transitional care programmes we will pay attention to the responsibilities of different stakeholders (ie, transitioning youth, their parents/caregivers, paediatric and adult physicians, and other healthcare and allied healthcare workers involved in delivering care) as well as various practical considerations about the timing, preparation and relational aspects of supporting the development of youths' autonomy and decision-making capacities. Moreover, we will search for hints in the literature about the processes through which preferences and values are solicited, and how services are delivered and evaluated. In doing so, we seek to reveal the implicit and explicit strategies that focus on patient and family values and preferences that may be embedded in transitional care programmes.

To our knowledge, no previous work has been carried out in a systematic way to map the explicit and implicit ways in which healthcare transition programmes address 
ethical issues. The objective of this project is to map implicit and explicit ethical issues within a body of empirical work on transitional care with youth with neurodisabilities in order to identify common themes, promising strategies and knowledge gaps. A scoping review of this kind has the potential to reveal hidden ethical issues in the delivery of transitional care services that may undermine the goals of the programme such that adolescents and young adults and their families leave paediatric services with little support to guide the next steps of their care. It can also provide valuable information about how certain visions and practices may challenge or facilitate a successful transition that respects the values and preferences of young adults with neurodisabilities.

\section{METHODS AND ANALYSIS}

\section{Scoping review protocol design}

For this review, a methodological framework for scoping reviews will be applied. ${ }^{12-15}$ Our guiding definition for this scoping review follows Colquhoun et al, ${ }^{15}$ who propose that scoping reviews are 'a form of knowledge synthesis that addresses an exploratory research question aimed at mapping key concepts, types of evidence, and gaps in research related to a defined area or field by systematically searching, selecting, and synthesizing existing knowledge' (p 4). In line with common goals of scoping reviews, this review has been designed 'to examine the extent, range and nature of research activity; this type of review might not describe research findings in any detail but is a useful way of mapping fields of study where it is difficult to visualize the range of material that might be available' ( $p$ 21). ${ }^{13}$ Moreover, as one of our main goals is to develop a repository of knowledge that can be useful for clinicians and programme managers in transitional care, we appreciate that scoping reviews are useful for summarising and disseminating research findings to policymakers, practitioners and consumers.

Following Arksey and O'Malley's foundational framework, our work will consist of the following main stages: (A) identifying the research question; (B) identifying relevant studies; (C) study selection; (D) charting the data; and (E) collating, summarising and reporting the results. Finally, we will incorporate a sixth optional step ${ }^{14}$ as preliminary findings will be shared with stakeholders for the purposes of consultation, knowledge transfer and exchange.

\section{Stage 1: identifying the research question}

The preliminary research questions listed below provide an initial roadmap for the stages that follow and will guide this scoping review:

1. What ethical values and principles are explicitly or implicitly embedded in the vision of transitional care programmes (eg, what guiding values, goals and principles are embedded in transitional care programmes that have ethical implications or that address ethical issues)?
Box 1 Terms selected for the development of the search filters by category

MeSH terms for main concepts

continuity of patient care [MeSH Terms]; transition to adult care [MeSH Terms]; adolescent [MeSH Terms]; ethics [MeSH Terms]; personal autonomy [MeSH Terms]; patient preference [MeSH Terms]; patient participation [MeSH Terms]

\section{Non-MeSH terms}

continuity of care [Text Word]; transitional care [Text Word], transition to adult care [Text Word]; autonomy [Text Word]; preference [Text Word]; self-determination [Text Word]; empower [Text Word]; ethic* [Text Word]; youth [Text Word] adolesc* [Text Word]

\section{Other MeSH terms}

ethics [MeSH Subheading]

\section{Combination of MeSH terms with other terms}

(/(()(((continuity of patient care [MeSH Terms]) OR continuity of care [Text Word]) $O R$ transitional care Text Work)) $O R$ transition to adult care [Text Word]) OR "transition to adult care" [MeSH Terms])) AND (((((adolescent [MeSH Terms]) AND youth [Text Word]) AND adolesc*)) AND (((()(continuity of patient care [MeSH Terms]]) OR continuity of care [Text Word]) OR transitional care)) OR transition to adult care [Text Word]) OR "transition to adult care" [MeSH Terms]))) AND ((()((()((ethics [MeSH Subheading]) OR ethics [MeSH Terms]) OR "personal autonomy" [MeSH Terms]) OR "patient preference" [MeSH Terms]) OR patient participation [MeSH Terms]) OR autonomy [Text Word]) OR preference [Text Word]) OR self-determination [Text Word]) OR empower [Text Word]) OR ethic*[Text Word])

2. What ethical values and principles are embedded in service delivery guidelines of transitional care programmes (eg, what ethical principles are described in the process of delivery of service)?

3. Which ethical issues have been encountered in transitional care?

4. What strategies have been employed to recognise and address ethical issues in transitional care?

5. What key policy and practice recommendations can be identified from this knowledge synthesis?

\section{Stage 2: identifying relevant studies}

To develop our search strategy, we consulted with a librarian at McGill University who has specialised expertise and a publication record on knowledge synthesis methods. The search strategy was developed based on keywords and index terms related to transitional care programmes and ethics in PubMed based on Medical Subject Headings (MeSH) terms (see box 1).

A literature search will be conducted to identify relevant studies published in English and French published between the years 1980 and 2018 and focused on paediatric to adult healthcare transitional care programmes. Studies will be identified through a search of academic databases including PsycINFO, CINHAL (Cumulative Index to Nursing and Allied Health Research), Ovid EMBASE (Excerpta Medica Database), ISI Web of Science, Ovid MEDLINE and Scopus. Keywords and index terms 
will be adapted to match naming conventions of various electronic databases.

\section{Stage 3: study selection}

Studies will be imported from the scholarly databases into EndNote and into an MS Excel file and will undergo two levels of screening. The first level of screening (initial screening) will consist of a review of study titles and abstracts and will be performed by a research assistant using Rayyan, ${ }^{16}$ a web and mobile application for systematic reviews. Inclusion criteria for the initial screening will be as follows: (A) the study is about paediatric to adult healthcare transition; (B) the paper is a full report (opinions, editorial, conference abstract and reviews to be excluded); (C) the study is about youth and adolescents, their caregivers and members of their families, healthcare providers, programme managers and policymakers; (D) published in English or French. Each article will be coded: (0) exclude; (1) include; or (2) unclear. If a study is coded as unclear from the study title and abstract, the first author and research assistant will try to resolve the coding by consensus. If disagreement persists, the last coauthor (ER) will weigh in.

\section{Study selection method}

Full-text articles will be assessed for eligibility in the scoping review. The first author and a research assistant will conduct screening of full-text articles. Discrepancies will be resolved by consensus. If disagreement persists, the last coauthor (ER) will weigh in.

\section{Stage 4: charting the data \\ Conceptual framework}

Prior to commencing the proposed scooping review, we developed a framework, entitled 'Integrative Framework of Ethics in Transitional Care' (online supplementary file), through a dedicated content extraction strategy to serve as a point of departure and a guide for the coding and mapping of ethical principles related to transitional care, tensions that may emerge in practice and proposed strategies and/or duties to address challenges in practice. The framework synthesises fundamental challenges identified in the literature on ethics in transitions from paediatric to adult healthcare and serves to guide the extraction of data in studies in order to: (1) identify and characterise ethical principles in transitional care based on empirical studies on transition programme, and (2) identify and characterise ethical tensions that may emerge in transitional care programmes and documented examples of suggested strategies to address those. This framework will be iteratively refined and further developed as we conduct the scoping review.

A data extraction form, developed in Microsoft Excel, will be used to chart key study information and relevant content. Two reviewers will test the utility of the form on a random sample of 10 studies and the form will be revised iteratively. We will record bibliographic information (eg, author(s); year of publication; origin/country of study) and study characteristics (eg, aims/purpose of the study; study population; methods/methodology; outcomes and details of outcome (eg, measures)). We will also record additional information about aspects of the study design related to stakeholder involvement in the research design. Guided by the Integrative Framework of Ethics in Transitional Care Programs, we will record and classify key information that relates to the scoping review questions: (1) key ethical principles embedded in the vision of the programmes (see guiding conceptual framework on ethical principles relevant to healthcare transition programmes); (2) key ethical principles embedded in service delivery guidelines; (3) key ethical issues reported/encountered by healthcare professionals; (4) key strategies developed to address ethical issues, and (5) key policy and practice recommendations. We will continue to build and refine the framework through the data extraction/collection process and stakeholder engagement.

\section{Stage 5: collating, summarising and reporting the results}

Results of the literature search and study screening will be presented in a flow chart that will outline the review decision process. ${ }^{17}$ The study characteristics outlined above will be presented both in diagrammatic/tabular and narrative form. Key findings related to the research questions will be organised by key themes, trends or patterns following the principles associated with the refined conceptual framework.

\section{Stage 6: consultation}

\section{Patient and public involvement}

The development of the research questions for this scoping review did not involve patients or the public. However, consultation is an integral part of this project. In their original methods paper, Arksey and O'Malley ${ }^{13}$ suggest that consultation is an optional stage in the scoping review process; however, in Levac et als $\mathrm{s}^{14}$ study this final stage is considered to be a valuable knowledge transfer mechanism. As the goal of our work is to identify gaps in service delivery and to recommend practice improvement, we will include this stage in the review to ensure that findings from our review are presented in such a way that they have clinical usefulness and relevance. Outreach efforts will be made to develop an advisory/consultation group comprising key stakeholders including people with lived experience of transitions such as youth in transition, their parents, researchers, clinicians and programme managers and policymakers.

\section{DISSEMINATION}

Scoping review results (both preliminary and final) will be disseminated in peer-reviewed publications but will also be presented at key conferences such as the annual meetings of the Canadian Bioethics Society, the American Academy of Cerebral Palsy and Developmental Medicine and the American Society for Bioethics and Humanities. We will also seek out other opportunities to discuss this project and its preliminary findings 
with partners in the paediatric setting, adult healthcare and rehabilitation communities comprising young adults, families, clinicians, programme manager and researchers.

\section{CONCLUSION}

The improvement of transitional care delivery has been identified as a focal point among paediatric healthcare service providers and administrators, given what is at stake for maintaining the health and well-being of youth in their care. Within the burgeoning literature documenting processes and experiences of transitional care, limited attention has been paid to the ethical issues encountered by healthcare providers, youth and their caregivers during this period of intense change and shifting responsibilities. This is surprising given the significant social and ethical implications that transitions into adulthood represent for the emerging autonomy and moral agency of youth with neurodisabilities and their families. The transition of healthcare also has implications on principles of trust between service users that are important to nurture when changing service providers.

We propose a protocol to undertake a scoping review of the literature on transitional care with an eye to, notably, document the ethical principles embedded in the vision of transitional care programmes, the ethical issues encountered in transitional care and the strategies employed to recognise and address these ethical issues. The resulting review should highlight these important aspects of clinical practice and health service delivery, ideally yielding a preliminary, literature-based, repository of policy and practice recommendations that can be of use to researchers and health system administrators. In subsequent phases of our research, we will use the evidence gathered in this review to develop ethics-informed tools and then test their usability and utility in a participatory action research project in collaboration with key stakeholders.

\section{Author affiliations}

${ }^{1}$ Pragmatic Health Ethics Research Unit, Institut de recherches cliniques de Montréal, Montreal, Quebec, Canada

${ }^{2}$ Faculté des études supérieures et postdoctorales (Programme de sciences neurologiques), Université de Montréal, Montréal, Quebec, Canada

${ }^{3}$ Department of Pediatrics, McMaster University, Hamilton, Ontario, Canada ${ }^{4}$ CanChild Centre for Childhood Disability Research, Institute for Applied Health Sciences, McMaster University, Hamilton, Ontario, Canada

${ }^{5}$ Département de médecine et Département de médecine sociale et préventive (Programmes de bioéthique), Université de Montréal, Montreal, Quebec, Canada ${ }^{6}$ Division of Experimental Medicine (Biomedical Ethics Unit), Department of Neurology and Neurosurgery, McGill University, Montréal, Québec, Canada

Acknowledgements Genevieve Gore, Liaison Librarian (McGill University), provided expertise on search terms and literature search strategies. Audrey Francoeur, Research Assistant (Pragmatic Health Ethics Research Unit, Institut de recherches cliniques de Montreal), tested article screening and data extraction procedures. Matthew Sample and Ariane Quintal (Pragmatic Health Ethics Research Unit, Institut de recherches cliniques de Montreal) provided conceptual feedback on the conceptual model represented in figure 1.
Contributors Aline Bogossian, Eric Racine and Jan Willem Gorter devised the project, the main conceptual ideas and the design of the protocol. Aline Bogossian and Eric Racine drafted the manuscript and Jan Willem Gorter commented on the manuscript. Aline Bogossian, Eric Racine and Jan Willem Gorter conducted revisions in the manuscript of important intellectual content and approved the version to be published.

Funding This work was supported by a postdoctoral award from the Kids Brain Health Network and a Canadian Institutes for Health Research Career Award, both held by ER. JWG holds the Scotiabank Chair in Child Health Research.

Competing interests None declared.

Patient consent Not required.

Provenance and peer review Not commissioned; externally peer reviewed.

Open access This is an open access article distributed in accordance with the Creative Commons Attribution Non Commercial (CC BY-NC 4.0) license, which permits others to distribute, remix, adapt, build upon this work non-commercially, and license their derivative works on different terms, provided the original work is properly cited, appropriate credit is given, any changes made indicated, and the use is non-commercial. See: http://creativecommons.org/licenses/by-nc/4.0/.

\section{REFERENCES}

1. Palisano RJ, Di Rezze B, Stewart D, et al. Life course health development of individuals with neurodevelopmental conditions. Dev Med Child Neurol 2017;59:470-6.

2. Gorter JW, Stewart D, Smith MW, et al. Pathways toward positive psychosocial outcomes and mental health for youth with disabilities: A knowledge synthesis of developmental trajectories. Can J Commun Ment Health 2014;33:45-61.

3. Morris C, Janssens A, Tomlinson R, et al. Towards a definition of neurodisability: A Delphi survey. Dev Med Child Neurol 2013;55:1103-8.

4. Blum RW, Garell D, Hodgman $\mathrm{CH}$, et al. Transition from childcentered to adult health-care systems for adolescents with chronic conditions. A position paper of the Society for Adolescent Medicine. J Adolesc Health 1993;14:570-6.

5. CAPHC Community of Practice in Transition. A guideline for transition from paediatric to adult health care for youth with special health care needs: A national approach. CAPHC Community of Practice in Transition: Ontario, 2016.

6. Nguyen T, Stewart D, Gorter JW. Looking back to move forward: Reflections and lessons learned about transitions to adulthood for youth with disabilities. Child Care Health Dev 2018;44:1-6.

7. Racine E, Bell E, Yan A, et al. Ethics challenges of transition from paediatric to adult health care services for young adults with neurodevelopmental disabilities. Paediatr Child Health 2014;19:65-8.

8. Suris JC, Akre C. Key elements for, and indicators of, a successful transition: An international Delphi study. J Adolesc Health 2015;56:612-8.

9. Campbell F, Biggs K, Aldiss SK, et al. Transition of care for adolescents from paediatric services to adult health services. Cochrane Database Syst Rev 2016;4:CD009794.

10. Kaufman $\mathrm{H}$, Horricks L, Kaufman M. Ethical considerations in transition. Int J Adolesc Med Health 2010;22:453-9.

11. Ronen GM, Rosenbaum PL. Reflections on ethics and humanity in pediatric neurology: The value of recognizing ethical issues in common clinical practice. Curr Neurol Neurosci Rep 2017;17:1-8.

12. Grant MJ, Booth A. A typology of reviews: An analysis of 14 review types and associated methodologies. Health Info Libr J 2009;26:91-108.

13. Arksey H, O'Malley L. Scoping studies: Towards a methodological framework. Int J Soc Res Methodol 2005;8:19-32.

14. Levac D, Colquhoun H, O'Brien KK. Scoping studies: Advancing the methodology. Implement Sci 2010;5:69.

15. Colquhoun HL, Levac D, O'Brien KK, et al. Scoping reviews: Time for clarity in definition, methods, and reporting. J Clin Epidemiol 2014;67:1291-4.

16. Ouzzani M, Hammady H, Federowicz Z, et al. Rayyan - A web and mobile app for systematic reviews Systematic Reviews2016;5:210.

17. Moher D, Liberati A, Tetzlaff J, et al. Preferred reporting items for systematic reviews and meta-analyses: The PRISMA statement. Annals of Internal Medicine 2009;15:264-9. 